\author{
Marquette University \\ e-Publications@Marquette
}

College of Nursing Faculty Research and

Publications

Nursing, College of

6-2005

\title{
Mentoring Staff Members as Patient Safety Leaders: The Clarian Safe Passage Program
}

Kathryn Rapala

Marquette University, kathryn.rapala@marquette.edu

Follow this and additional works at: https://epublications.marquette.edu/nursing_fac

Part of the Nursing Commons

\section{Recommended Citation}

Rapala, Kathryn, "Mentoring Staff Members as Patient Safety Leaders: The Clarian Safe Passage

Program" (2005). College of Nursing Faculty Research and Publications. 796.

https://epublications.marquette.edu/nursing_fac/796 


\title{
Marquette University
}

\section{e-Publications@Marquette}

\section{Nursing Faculty Research and Publications/College of Nursing}

This paper is NOT THE PUBLISHED VERSION; but the author's final, peer-reviewed manuscript. The published version may be accessed by following the link in the citation below.

Critical Care Nursing Clinics of North America, Vol. 17, No. 2 (June 2005): 121-126. DOI. This article is (C) Elsevier and permission has been granted for this version to appear in e-Publications@Marquette. Elsevier does not grant permission for this article to be further copied/distributed or hosted elsewhere without express permission from Elsevier.

\section{Mentoring Staff Members as Patient Safety Leaders: The Clarian Safe Passage Program}

\author{
Kathryn Rapala \\ Clarian Health Partners, Indianapolis, IN
}

We are at a crossroad in patient safety. Patient safety definitions, research, products, and implementation strategies have become as complex as health care itself. Almost every professional and regulatory organization now has a position or strategy to address the estimated 44,000 to 98,000 deaths per year due to health care error [1]. Memorizing and implementing the Joint Commission for Accreditation National Patient Safety Goals is not enough to establish and maintain a culture of safety. Patient safety literature offers few practical solutions on the implementation of an integrated, system wide application of patient safety approaches to clinical practice. It is our responsibility to teach those who provide direct patient care the concepts of patient safety and to provide a knowledge management network to support patient safety knowledge. In turn, the frontline practitioners can teach us about the patient safety challenges they face on a daily basis. Only then can we solve frontline patient safety issues.

The Clarian Safe Passage ${ }^{1}$ Program, in which trained staff nurses serve as the unit patient safety experts, in concert with the American Association of Critical Care Nurses (AACN) Synergy Model of 
Patient Care, have provided a patient safety knowledge and mentoring program that gives staff nurses the skills and tools to identify and address patient safety issues on a day-to-day basis.

\section{Patient safety methodology}

The ultimate goal of patient safety is to produce reliable health care. Reliability is the measurable capability of a process or procedure to perform its intended function in the required time under commonly and uncommonly occurring conditions [2]. High reliability organizations are those in which error seldom occurs even in dangerous environments. Examples of high reliability organizations include nuclear power plants and aircraft carriers. In contrast to high reliability organizations, which generally excel at one core process, health care is a highly complex environment based on individual patient and provider characteristics.

Five characteristics of high reliability organizations must be the foundation for health care patient safety improvement. The first is preoccupation with failure, where any near miss or error is examined with all the attention of a sentinel event. The second is the reluctance to simplify, where the entire complex work process is a part of any decision. Minority reports are welcome and necessary for the overall organizational picture, and oversimplification in summary is not allowed. The third characteristic of a high reliability organization is sensitivity to operations. The high reliability organization focuses attention on the front line, where gaps and latent failures are proactively identified before escalation into error. This characteristic requires frontline workers to speak up to voice concerns in a nonpunitive environment, and for the organization to foster the culture of safety. Commitment to resilience, or the ability of staff and the organization to recover from error, is the fourth characteristic. Finally, and perhaps most importantly, high reliability organizations exhibit deference to expertise. Critical decisionmaking occurs at the level where the expertise of the organization exists. This frontline problem solving also includes the ability to raise questions and issues to higher levels of the organization, including those in a position of higher authority [3]. The Safe Passage Program operationalizes and provides continuous feedback regarding these characteristics.

\section{Synergy: providing safe passage through the health care system}

The Synergy Model of patient care, jointly developed by the AACN and Clarian, is in essence the creation of a high reliability infrastructure designed to produce consistently reliable patient outcomes. In the Synergy Model, patient characteristics - stability, complexity, predictability, resiliency, vulnerability, participation in decision-making and resource availability-drive nursing competencies. Nursing competencies are clinical judgment, advocacy and moral agency, caring practices, facilitation of learning, collaboration, systems thinking, diversity of responsiveness and clinical inquiry. When patient and nursing competencies match, the result is optimal care for the patient. According to Dr. Martha Curley, "clinical expertise, that is skilled clinical knowledge, discretionary judgment, and the ability to integrate complex multisystem effects and understanding the trajectory of illness and human response to illness, creates safe passage for the patient" [4]. Safe passage for the patient is the ultimate goal of the Synergy Model of patient care: moving the patient through the health care system with reliable and good outcomes.

The Clarian Safe Passage Program has been developed to foster clinical judgment and systems thinking of the staff nurse in relation to safe patient care. Based on the concept that frontline staff has the best 
understanding of work at the sharp end of practice, Safe Passage is a staff driven model. The incorporation of Safe Passage throughout the organization, coupled with other patient safety initiatives, has positively impacted the culture of patient safety within Clarian and given frontline staff the knowledge, the forum, and the voice to proactively prevent or to mitigate patient error.

\section{Safe passage curriculum}

Every person who works or volunteers in health care has an obligation to practice patient safety citizenship. Baseline patient safety citizenship includes helping, voice, stewardship, whistle blowing, keeping informed, and initiating safety related change [5]. The Safe Passage Program incorporates patient safety citizenship into professional behavior by providing a patient safety curriculum for staff based on the high reliability concepts outlined above. The curriculum includes content that provides a basic grounding in the following patient safety methodologies. It is then expected that Safe Passage nurses will mentor patient safety citizenship within the system.

\section{Work complexity}

Understanding work complexity is perhaps the single most important concept for the Safe Passage nurses to master. Work constantly changes based on a constellation of patient and nursing characteristics, volumes, and resource allocation. Gaps and resulting error occur when organizational defenses such as policies or procedures do not align with the complex work environment. The most popular visual concept in Safe Passage is Emily Patterson's timeline illustration of a nurse passing medications that records the many interruptions in the act of hanging an intravenous fluid bag (see Fig. 1). Understanding work complexity provides the basic building block of a nonpunitive environmentanalyzing error from a systems rather than a blame perspective.

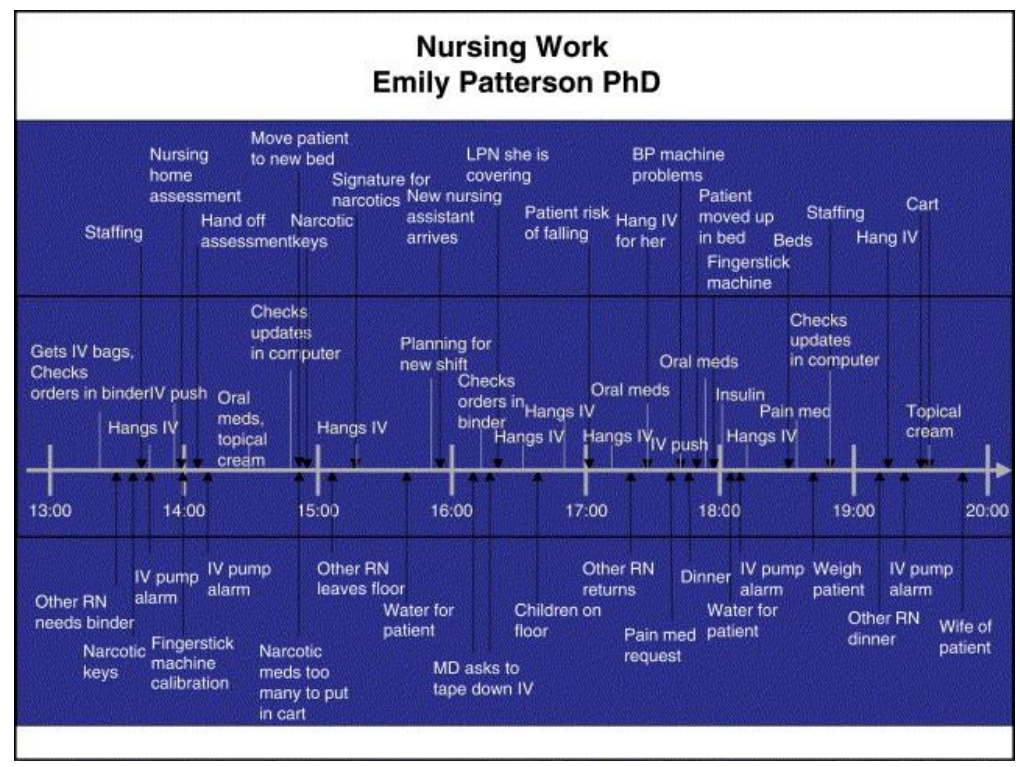

Fig. 1. Timeline illustration of a nurse passing medications that records the many interruptions in the act of hanging an intravenous fluid bag. (Courtesy of Emily Patterson, PhD, VA Midwest Patient Safety Center of Excellence.) 


\section{"First Story/Second Story"}

First Story Second Story is actually cognitive task analysis, an enhanced root cause analysis refined by Gary Klein [6]. This concept allows Safe Passage nurses to apply work complexity and systems level thinking to any error. Instead of writing "incorrect IV fluid hung" on an occurrence report, Safe Passage nurses are trained using this technique to focus on why the incorrect IV fluid was hung, uncovering gaps and latent failures in the system. Safe Passage nurses practice this technique on near misses, where an error has been caught before it reaches the patient, to avoid coworkers feeling blamed in the analysis.

\section{Proactive patient safety}

The Safe Passage nurse is also encouraged to proactively identify risks and prevent error, especially in relation to the introduction of new products or processes. Three questions are asked: How will this change my work? If the process will fail, why? What can we do to prevent such a failure? Members are encouraged to identify work-arounds that affect patient safety before an error occurs.

\section{Knowledge management and mentoring structure}

The Safe Passage Program started with a small core group of willing staff that was taught basic patient safety curriculum. Although our early innovators thought the content was interesting, the concepts did not disseminate easily. A knowledge management structure was needed. In fact, the Institute of Medicine has stated that high reliability organizations use knowledge management practices to create a learning organization, and that this principle is not consistently applied in the work environment of nurses.

Safe Passage nurses participate in a Clarian system-wide monthly meeting. This meeting provides an opportunity to share experiences and issues across units and departments. Each Safe Passage nurse also participates in a local monthly Safe Passage Council meeting. Safe Passage Councils include Operating Room, Mother/Baby, Emergency and Trauma Center, Pediatric, and Multi-specialty and Intensive Careeducators, managers, and frontline staff facilitate the Safe Passage Council.

The tone of the Safe Passage Councils is important. To foster discussion and dialogue, ideas and observations of the Safe Passage nurses are encouraged. Comments, most often from visitors or those outside the council, such as, "that's the way it is here on this unit-it will never change," or, "everyone's tried to fix that issue and nothing has worked-just give up," must not be allowed to end a discussion, observation, or suggestion. Meetings are not for management, but for patient safety dialogue and nontraditional thinking to mitigate error. However, the link to operations and the support of administration are key elements of the Safe Passage Program.

Other Safe Passage knowledge management practices designed to disseminate knowledge include:

- The Safe Passage newsletter

- The SEE (Sentinel Event Extra), a one-page alert distributed to notify units of internal and external patient safety issues

- The Safe Passage Manual, a binder located on each unit that includes occurrence report information, Institute for Safe Medical Practices newsletters, the Safe Passage newsletter, and other presentations or reminders ( Safe Passage members manage the binders) 
- A Safe Passage email distribution list

- A link to operations through clinical practice groups, unit meetings, managers and directors meetings

- Regular reports to the Clarian Board of Directors.

\section{Safe passage as a mentoring program}

Internal mentoring

Once the core curriculum is taught, Safe Passage nurses as unit experts will face inevitable organizational challenges. It is up to peers, as well as operations managers, to support these challenges. One local Safe Passage Council had talked quite extensively about nonpunitive reporting and how to analyze patient error. This particular council had a culture where the perception was that the staff member who made the error was at fault. A manager brought an issue to the Safe Passage Council for review, and stated that it was a compliance issue and that staff that did not meet the standard would be disciplined. The Safe Passage Council correctly discussed the systemic reasons for failure of the process, and made several recommendations to the manager. After the meeting, a member stated that if the Safe Passage Council had not "walked the talk," he would have left the room. This Safe Passage nurse is now one of the strongest patient safety advocates in this area.

Once issues come forward in Safe Passage by members, discussion and action must be taken. Identified patient safety issues may be correctable on the unit, at the departmental level, or may require systemic intervention. To obtain frontline input, equipment, product, and process changes are brought directly to the monthly Safe Passage meeting for recommendations.

\section{Mentoring among safe passage members}

The Safe Passage infrastructure allows members to mentor each other, as well as other coworkers. The monthly agendas are structured so that Safe Passage nurses working on projects may share them with the system-wide group. This presentation serves two purposes. First, it shares patient safety knowledge on a system level. Second, the presentation allows Safe Passage nurses to use PowerPoint and speak in a public forum. This is a skill that many frontline staff find daunting, but these presentations to peers promote professional development.

Safe Passage nurses mentor other staff as well. Two adult critical care Safe Passage members did a journal club presentation on Safe Passage including two patient safety studies. The attendance set a new record: close to 200 staff members attended during several presentation times. An emergency room Safe Passage nurse compiled "First Story/Second Stories" on several patient events and presented them to her unit during popular in-service sessions. Self-motivated staff-to-staff mentoring, disseminates information more quickly to many more individuals.

\section{External mentoring}

Safe Passage nurses are mentored by external sources as well. The Safe Passage Program sponsors members to attend key educational conferences such as the National Patient Safety National 
Conferences. Because Safe Passage is a staff driven program, Safe Passage nurses have participated in national forums and panels.

Additionally, nationally known patient safety experts have made key contributions to to the Safe Passage Program. One of the most important external mentors has been a faculty member from the Indiana University School of Nursing. Not only has Dr. Patricia Ebright helped develop the Safe Passage Program, she assists with the curriculum, and actively mentors the Safe Passage members. She is a "safe" and objective person to discuss issues with-one who is not operationally responsible for any Clarian staff members, but who can provide patient safety guidance.

\section{Safe passage mentoring as a patient safety check and balance}

The concept of the Safe Passage nurse requires staff nurses to identify patient safety issues on the unit. These patient safety issues may have been present on the unit for some time, or may represent a particular error such as a sentinel event. Since the Safe Passage nurse is taking a fresh look at patient safety on the unit, traditional organizational barriers can sometimes impede the information transfer. The Safe Passage Program provides a mentoring process to ensure and protect information flow from the front line. For example, a critical care Safe Passage nurse correctly identified a patient safety event that started on one unit and ended on the critical care unit. When the Safe Passage nurse identified the issue, the perception was that the event occurred on the first unit, so it should have been reported by that unit. However, staff members on the first unit were not aware of the outcome. The Safe Passage nurse sought guidance within the mentoring structure, which confirmed that the event should be reviewed as a systems issue. Without the mentoring aspect of Safe Passage, the opportunity for improvement could have been lost.

\section{Common themes provide mentoring opportunities}

As the Synergy Model of Care and the Safe Passage Program mature, common themes from both programs have emerged. Validated by themes in the root cause analysis work at Clarian, collaboration, intuition, rescue, and communication have arisen as areas of challenge within the organization. These common areas are important for several reasons. First, common themes represent the leap from counting errors to identifying and addressing the root causes of errors. Second, it allows the organization to effectively focus resources to coordinate educational, process improvement, and other actions to address the root causes. Finally, multiple sources of information serve to validate and confirm these systems issues.

Based on these common themes, Safe Passage has created a mentoring process regarding intuition. Intuition occurs when a staff member knows something or anticipates how a situation is going to unfold, but the staff member cannot articulate how he or she knows. Experts interpret the overall clinical picture by spotting leverage points, forming expectancies, projecting the future, detecting anomalies, performing workarounds and making time and distance judgments. However, the expert does this unconsciously, making it very difficult to transfer this knowledge to the novice. Novice staff members, in contrast, look at pieces of the process and rely on routines in patient care. This fragmented assessment creates gaps in care and patient safety issues [6]. The key is to ask certain questions as events unfold to validate care. This approach also uses methods for team validation and handoffs. 


\section{Safe passage outcomes: measures of success}

Safe Passage Program outcomes may be measured in several different ways. From an evidence-based practice standpoint, a safe health care environment is a learning organization. To become a learning organization, an organization must take advantage of all sources of knowledge, use systemic experimentation to generate new knowledge internally, and transfer knowledge quickly and efficiently throughout the organization [7]. The Safe Passage Program has created an infrastructure to support this evidence-based practice.

The Leapfrog Group, a group of employers that has focused efforts on patient safety improvement, has recently adopted the National Quality Forum Evidenced-Based Safe Practices. The most heavily weighted measure in this model is the culture of safety. The measure requires patient safety information flow to the front line, as well as a commitment to the culture of safety [8].

The Safe Passage emphasis on a nonpunitive patient safety environment has produced significant cultural changes. In concert with the root cause analysis structure, occurrence reporting (including near miss reporting), has increased dramatically in one year. Safe Passage nurses are encouraged to participate in the root cause analyses on their units, providing patient safety expertise and peer support. Though Safe Passage is not the sole reason for these measures, the culture shift and mentoring as a result of Safe Passage has positively contributed to the overall patient safety environment.

Safe Passage members are identifying and addressing patient safety issues on a unit basis. These proactive interventions are termed "good catches," and are occurring more frequently as the Safe Passage Program matures. Safe Passage nurses are reaching across traditional boundaries to effect system changes.

\section{Summary}

The Safe Passage Program creates an effective knowledge management structure for patient safety, allowing information to flow to the units and back to administration. The Safe Passage Program allows us to listen to the frontline caregivers. Rather than delivering a lecture or a once-a-year in-service, the Safe Passage Program has created a continual patient safety mentoring program. Mentoring ensures successful knowledge transfers by allowing staff nurses to develop patient safety expertise.

Safe Passage enhances the Synergy Model of Patient Care nurses' competencies, especially critical judgment, collaboration, systems thinking, and clinical inquiry. Developing these strengths allows the Safe Passage nurse to mentor others, creating safe passage for patients through the system. This fundamental redesign of the nursing process in concert with an emphasis on patient safety has provided the foundation for reaching high reliability in health care.

\section{References}

[1] Institute of Medicine Report. L. Kohn, J.M. Corrigan, M.S. Donaldson (Eds.), To err is human: building a safer health system, National Academy Press, Washington, DC (1999), pp. 1-287

[2] D. Berwick, T. Nolan. High reliability health care. Available at http://www.ihi.org/IHI/Topics/Reliability/ReliabilityGeneral/EmergingContent/HighReliabilityHe althCarePresentation.htm. Accessed October 2004 
[3] K.E. Weick, K.M. Sutcliffe. Managing the unexpected-assuring performance in an age of complexity, Jossey-Bass, San Francisco (CA) (2001)

[4] M.Q. Curley. Patient-nurse synergy: optimizing patients outcomes. Am J Crit Care, 7 (1) (1998), pp. 64-72

[5] D.A. Hoffman, F.P. Morgeson, S.J. Gerras. Climate as a moderator of the relationship between leader-member exchange and context specific citizenship: safety climate as an exemplar. $J$ Appl Psychol, 88 (1) (2003), pp. 170-178

[6] G. Klein. Intuition at work-why developing your gut instincts will make you better at what you do, Currency Books, New York (2003), pp. 1-307

[7] A. Page (Ed.), Keeping patients safe-transforming the work environment, The National Academy Press, Washington, DC (2004), p. 108

[8] The National Quality Forum. Safe practices for better healthcare, The National Quality Forum, Washington, DC (2003), pp. 1-200

${ }^{1}$ The Safe Passage (C term and process is proprietary to Clarian Health Partners, Inc., 2002; all rights reserved; patent pending. 